\title{
AN EFFECTIVE ANALYSIS OF THE DENJOY RANK
}

\author{
LINDA BROWN WESTRICK
}

\begin{abstract}
We analyze the descriptive complexity of several $\Pi_{1}^{1}$ ranks from classical analysis which are associated to Denjoy integration. We show that $V B G, V B G_{*}, A C G$ and $A C G_{*}$ are $\Pi_{1}^{1}$-complete, answering a question of Walsh in case of $A C G_{*}$. Furthermore, we identify the precise descriptive complexity of the set of functions obtainable with at most $\alpha$ steps of the transfinite process of Denjoy totalization: if $|\cdot|$ is the $\Pi_{1}^{1}$-rank naturally associated to $V B G, V B G_{*}$ or $A C G_{*}$, and if $\alpha<\omega_{1}^{c k}$, then $\{F \in C(I):|F| \leq \alpha\}$ is $\Sigma_{2 \alpha}^{0}$-complete. These finer results are an application of the author's previous work on the limsup rank on well-founded trees. Finally, $\left\{(f, F) \in M(I) \times C(I): F \in A C G_{*}\right.$ and $F^{\prime}=f$ a.e. $\}$ and $\{f \in M(I): f$ is Denjoy integrable $\}$ are $\Pi_{1}^{1}$-complete, answering more questions of Walsh.
\end{abstract}

Real analysis of the early 20th century featured a number of naturally occuring $\Pi_{1}^{1}$ complete sets. The most prominent example may be the set of differentiable functions on the unit interval $I$, considered as a subset of $C(I)$, the metric space of continuous functions on $I$ with the supremum norm.

Any $\Pi_{1}^{1}$ set $A$ may be decomposed as a transfinite union $\cup_{\alpha<\omega_{1}} A_{\alpha}$, where each $A_{\alpha}$ is Borel. When this is done in a sufficiently uniform way, the function which maps each $f \in A$ to the least $\alpha$ such that $f \in A_{\alpha}$ is called a $\Pi_{1}^{1}$-rank. Sometimes a $\Pi_{1}^{1}$ set has an obvious and natural $\Pi_{1}^{1}$-rank. This is the case for the set of well-founded trees $T \subseteq \omega^{<\omega}$ (the usual wellfounded tree rank), the collection of countable closed subsets of $I$ (the Cantor-Bendixson rank), and the collection of continuous functions obtainable by Denjoy totalization, as we shall see shortly. Other times, finding a rank that could be considered natural is more difficult. For example, in [KW86] Kechris and Woodin defined a suitably natural rank on the set of differentiable functions.

When the rank on $A$ is natural, it becomes meaningful to ask for the precise descriptive complexity of the initial segments $A_{\alpha}$. This was done implicitly for the well-founded tree rank in [GMS13], for the Cantor-Bendixson rank in [CM83] and [Lem87], and for the KechrisWoodin rank on differentiable functions in [Wes14]. The purpose of this paper is to show that the method used in Wes14 generalizes to give descriptive complexities for three hierarchies from classical analysis related to Denjoy totalization. We also give a new proof of a result obtained by both Cenzer and Mauldin [CM83] and independently Lempp [Lem87, concerning the descriptive complexity of the initial segments of the Cantor-Bendixson rank.

Denjoy totalization is a transfinite integration process developed by Denjoy in 1912 to solve the problem of recovering $F$ from $F^{\prime}$ whenever $F$ is an everywhere differentiable function in $C(I)$. The process does a little more, recovering also some a.e. differentiable $F$, but not all of them. The set of $F \in C(I)$ recoverable from $F^{\prime}$ by Denjoy totalization is denoted $A C G_{*}$. The related sets $A C G, V B G_{*}$ and $V B G$ are described in the next section.

The main result is the following. Let $|\cdot|_{V B},|\cdot|_{V B_{*}},|\cdot|_{A C}$ and $|\cdot|_{A C_{*}}$ denote the natural $\Pi_{1}^{1}$ ranks on $V B G, V B G_{*}, A C G$ and $A C G_{*}$ respectively.

The author was partially supported by the P.E.O. International Scholar Award. 
Theorem 1. Let $X=V B, V B_{*}$ or $A C_{*}$, let $Y \in 2^{\omega}$, let $1<\alpha<\omega_{1}^{Y}$, and let

$$
A_{\alpha}=\left\{F \in C(I):|F|_{X} \leq \alpha\right\} \text {. }
$$

Then $A_{\alpha}$ is $\Sigma_{2 \alpha}^{0}(Y)$, and for any $\Sigma_{2 \alpha}^{0}(Y)$ set $B$, there is a $Y$-computable reduction from $B$ to $A_{\alpha}$. In particular, $A_{\alpha}$ is $\Sigma_{2 \alpha}^{0}$-complete, and if $\alpha<\omega_{1}^{C K}$, then $A_{\alpha}$ is $\Sigma_{2 \alpha}^{0}$-complete.

Let $M(I)$ denote the Polish space of measurable functions on the unit interval, with metric given by $d(f, g)=\int_{I} \min (1,|f-g|)$. Our next theorem answers three questions in [Wal17].

Theorem 2. The following sets are all $\Pi_{1}^{1}$-complete:

(1) $V B G, V B G_{*}, A C G$ and $A C G_{*}$

(2) $\left\{(f, F) \in M(I) \times C(I): F \in A C G_{*}\right.$ and $F^{\prime}=f$ a.e. $\}$

(3) $\{f \in M(I): F$ is Denjoy integrable $\}$

The sets $\left\{F \in C(I):|F|_{X} \leq 1\right\}$ are better known as the collection of functions of bounded variation when $X=V B, V B_{*}$ and the collection of absolutely continuous functions when $X=A C, A C_{*}$. The following results filling in the $\alpha=1$ case of Theorem 1 have routine proofs but their statements may be of interest.

Theorem 3. (1) The set of continuous functions of bounded variation is $\Sigma_{2}^{0}$-complete.

(2) The set of absolutely continuous functions is $\Pi_{3}^{0}$-complete.

In Section 1, after some preliminaries we review the main tool in Wes14, an analysis of the descriptive complexity of the limsup rank on the set of well-founded trees. We also give the background on Denjoy totalization and the hierarchies $A C G_{*}, A C G, V B G_{*}$ and $V B G$. In Section 2 we apply this analysis to the simpler case of the Cantor-Bendixson derivative, recovering the aforementioned result of Cenzer, Mauldin, and Lempp. In Section 3 we prove hardness results for the hierarchies on $A C G_{*}, A C G, V B G_{*}$ and $V B G$, obtaining Theorem 2 and one direction of Theorem 1. In Section 4 we obtain matching descriptive results for all of these hierarchies except $A C G$, giving the other direction of Theorem 1. Section 5 contains open questions.

The author would like to thank Ted Slaman and Sean Walsh for a number of useful and interesting discussions, and the anonymous referee, whose comments have clarified the main arguments.

\section{Preliminaries}

1.1. Notation. We use standard computability-theoretic notation. Trees $T \subseteq \omega^{<\omega}$ can be encoded by elements of Cantor space. Let $\operatorname{Tr} \subseteq 2^{\omega}$ be the set of codes for such trees. Given $T \in T r$, we usually forget the encoding and treat $T$ as a subset of $\omega^{<\omega}$ anyway. The set $[T] \subseteq \omega^{\omega}$ is the set of paths through $T$. Let $\sigma^{\wedge} \tau$ denote the concatenation of $\sigma$ and $\tau$. If $\sigma \in T$, let $T_{\sigma}$ denote $\left\{\tau: \sigma^{\wedge} \tau \in T\right\}$, and for any $\sigma \in \omega^{<\omega}$, let $\sigma^{\wedge} T$ denote $\left\{\sigma^{\wedge} \tau: \tau \in T\right\}$. Trees of the form $T_{\sigma}$ where $\sigma=\langle n\rangle$ are commonly encountered, and for these trees we drop the brackets and just write $T_{n}$ instead of $T_{\langle n\rangle}$. Let $[\sigma]$ denote $\left\{X \in \omega^{\omega}: \sigma \prec X\right\}$ or $\left\{X \in 2^{\omega}: \sigma \prec X\right\}$, depending on the context. When a tree is in fact a subset of $2^{<\omega}$, we usually give it the name $S$, reserving $T$ for trees in Baire space.

The complement of a set $A \subseteq \omega^{\omega}$ is denoted $\neg A$. If $A, B, C, D \subseteq \omega^{\omega}$ and $f$ is a computable function from $A \cup B$ to $C \cup D$ with $f^{-1}(C)=A$ and $f^{-1}(D)=B$, we say that $f$ is a computable reduction from $(A, B)$ to $(C, D)$. If $B=\neg A$ and $D=\neg C$, we say $f$ is a computable reduction from $A$ to $C$. 
A tree $T \subseteq \omega^{<\omega}$ is well-founded, denoted $T \in W F$, if $[T]=\emptyset$. A set $A \subseteq \omega^{\omega}$ is $\Pi_{1}^{1}$ if there is a computable reduction from $A$ to $W F$. A $\Pi_{1}^{1}$ set $A$ is $\Pi_{1}^{1}$-complete if there is a computable function from $W F$ to $A$. The boldface notions $\boldsymbol{\Pi}_{1}^{1}$ and $\boldsymbol{\Pi}_{1}^{1}$-complete are obtained by replacing "computable" with "continuous", or equivalently, relativizing to an oracle.

For $n<\omega$, a set $X \in 2^{\omega}$ is $\Sigma_{n}^{0}$-complete if $X \equiv_{1} \emptyset^{(n)}$. To extend the notion of completeness to the ordinals, we assume some familiarity with hyperarithmetic theory: Kleene's $\mathcal{O}$, ordinal notations, the sets $H_{a}$ for $a \in \mathcal{O}$. For $a \in \mathcal{O}$, we use $|a|_{\mathcal{O}}$ to denote the ordinal coded by $a$. The supremum of ordinals represented in $\mathcal{O}$ is $\omega_{1}^{C K}$. Following [AK00], if $n \geq \omega$ we say that a subset $X \subseteq \omega$ is $\Sigma_{\alpha}^{0}$-complete if $X \equiv_{1} H_{2^{a}}$, where $a \in \mathcal{O}$ is any notation with $|a|_{\mathcal{O}}=\alpha$. All these notions can relativize to an oracle $Y \in 2^{\omega}$, using notation $\mathcal{O}^{Y},|a|_{\mathcal{O}}^{Y}, H_{a}^{Y}, \omega_{1}^{Y}, \Sigma_{\alpha}^{0}(Y)$.

These computability notions on subsets of $\omega$ can be extended to effective topological notions on subsets of $\omega^{\omega}$. A set $A \subseteq \omega^{\omega}$ is $\Sigma_{\alpha}^{0}(Y)$ if $\omega \leq \alpha<\omega_{1}^{Y}$ and there is an index $e_{0}$ and a notation $a \in \mathcal{O}^{Y \oplus \emptyset}$ such that for all $X \in \omega^{\omega}, a \in \mathcal{O}^{Y \oplus X}$ with $|a|^{Y \oplus X}=\alpha$ and

$$
X \in A \Longleftrightarrow e_{0} \in H_{2^{a}}^{Y \oplus X}
$$

If $\alpha<\omega$, replace $H_{2^{a}}^{Y \oplus X}$ with $H_{a}^{Y \oplus X}$. When showing that a set is $\Sigma_{\alpha}^{0}(Y)$, we usually use effective transfinite recursion. We assume familiarity with this method and sometimes avoid explicit mention of the sets $H_{2^{a}}^{Y \oplus X}$ while using it.

It is well-known that $\Sigma_{\alpha}^{0}=\bigcup_{\substack{Y \in 2^{\omega} \\ \alpha<\omega_{1}^{Y}}} \Sigma_{\alpha}^{0}(Y)$, where $\Sigma_{\alpha}^{0}$ refers to the $\alpha$ th level of the Borel hierarchy.

We use $I$ to refer to the unit interval, $C(I)$ for the space of continuous real-valued functions on $I$ with the supremum norm, and $M(I)$ for the space of measurable functions on the unit interval, with metric given by $d(f, g)=\int_{I} \min (1,|f-g|)$. In $C(I)$, the piecewise linear functions with rational endpoints form a countable dense subset; computing a element $F$ of $C(I)$ means providing, uniformly in $\varepsilon$, a piecewise linear function within $\varepsilon$ of $F$ in the supremum norm. In $M(I)$, the step functions with rational values and discontinuities at finitely many rational points form a countable dense subset, and can be used as the ideal points in a representation of $M(I)$ as an effectively presented metric space. Thus computing an element $f$ of $M(I)$ means providing, uniformly in $\varepsilon$, an ideal point of the type described above which is $\varepsilon$-close to $f$. A closed subset $P$ of $I$ is always coded by an enumeration of the open intervals $(p, q)$ with rational endpoints such that $[p, q] \cap P=\emptyset$.

1.2. Limsup rank. Our main tool is the limsup rank on well-founded trees, which was defined in Wes14 as follows.

Definition 1. Let $T \subseteq \omega^{<\omega}$ be a well-founded tree. If $T=\emptyset$, define $|T|_{l s}=0$. Otherwise, define

$$
|T|_{l s}=\max \left(\sup _{n}\left|T_{n}\right|_{l s},\left(\limsup \left|T_{n}\right|_{l s}\right)+1\right) .
$$

This rank is designed to line up nicely with Cantor-Bendixson type derivation processes in a way that will be explained below.

Theorem 4 ([Wes14]). For all constructive $\alpha>1,\left\{e: \phi_{e}\right.$ codes a tree $T$ with $\left.|T|_{l s} \leq \alpha\right\}$ is $\Sigma_{2 \alpha}$-complete.

The only reason for not allowing $\alpha=1$ in the above theorem is that it is $\Pi_{2}$ to tell whether $\phi_{e}$ is total, that is, whether it codes anything at all.

Almost for free, we can make topological claims in addition to computational ones. The next theorem follows from Theorem 4 by relativization. 
Theorem 5. For any nonzero $\alpha<\omega_{1}$, let

$$
A_{\alpha}=\left\{T \in \operatorname{Tr}:|T|_{l s} \leq \alpha\right\} .
$$

Then if $\alpha<\omega_{1}^{Y}$, we have $A_{\alpha} \in \Sigma_{2 \alpha}^{0}(Y)$, and for any set $B \in \Sigma_{2 \alpha}^{0}(Y)$, there is a $Y$-computable reduction from $B$ to $A_{\alpha}$. In particular, $A_{\alpha}$ is $\Sigma_{2 \alpha}^{0}$-complete, and if $\alpha<\omega_{1}^{C K}$, then $A_{\alpha}$ is $\Sigma_{2 \alpha}^{0}$-complete.

Proof. Let $a$ be a notation such that for all $X, a \in \mathcal{O}^{X \oplus Y}$ and $|a|_{\mathcal{O}}^{X \oplus Y}=2 \alpha$. For all $X$, by relativization we have

$$
H_{2^{a}}^{X \oplus Y} \equiv_{1}\left\{e: \phi_{e}^{X \oplus Y} \text { codes a tree } T \text { with }|T|_{l s} \leq \alpha\right\},
$$

where the pair of reductions witnessing the 1-equivalence do not depend on $X 11$ Letting $d_{0}$ be such that $\phi_{d_{0}}^{X \oplus Y}=X$, the reverse reduction in (11) provides $e_{0}$ such that for all $X$,

$$
X \in A_{\alpha} \Longleftrightarrow \phi_{d_{0}}^{X \oplus Y} \in A_{\alpha} \Longleftrightarrow e_{0} \in H_{2^{a}}^{X \oplus Y},
$$

so $A_{\alpha} \in \Sigma_{2 \alpha}^{0}(Y)$.

To show that a given $B \in \Sigma_{2 \alpha}^{0}(Y)$ can be $Y$-computably reduced to $A_{\alpha}$, it suffices to consider $B=\left\{X: n_{0} \in H_{2^{a}}^{X} \oplus Y\right.$ where $n_{0}$ is chosen to make $B \Sigma_{2 \alpha}^{0}(Y)$-universal. The forward reduction in (11) provides $m_{0}$ such that $X \in B \Longleftrightarrow \phi_{m_{0}}^{X \oplus Y} \in A_{\alpha}$, and the mapping $X \mapsto \phi_{m_{0}}^{X \oplus Y}$ is $Y$-computable.

1.3. Denjoy totalization. Classical real analysis includes the study of absolutely continuous functions, functions of bounded variation, and countable generalizations of these notions. We consider four classes of real-valued functions on $I$. They are $V B G$ (generalized bounded variation), $V B G_{*}$ (generalized bounded variation in the restricted sense), $A C G$ (generalized absolutely continuous) and $A C G_{*}$ (generalized absolutely continuous in the restricted sense).

To define these classes it is necessary to generalize the well-known definitions of bounded variation and absolute continuity to take into account also a closed set $E$ to which the function $F$ should be in some sense restricted. The non-asterisk definitions are the literal restrictions. The others take into account also the values of $F$ outside of $E$. The oscillation of a function $F$ on an interval $(a, b)$ is defined as $\omega(F, a, b)=\sup _{x, y \in(a, b)}|F(x)-F(y)|$.

Definition 2. Let $F \in C(I)$ and let $E \subseteq I$.

(1) We say $F$ is $V B$ (respectively $V B_{*}$ ) on $E$ if there is an $N$ such that for all nondecreasing sequences $a_{0}, b_{0}, \ldots a_{k}, b_{k} \in E$, we have $\sum_{i}\left|F\left(b_{i}\right)-F\left(a_{i}\right)\right|<N$ (respectively $\left.\sum_{i} \omega\left(F, a_{i}, b_{i}\right)<N\right)$.

(2) We say $F$ is $A C$ (respectively $A C_{*}$ ) on $E$ if for all $\varepsilon$ there is a $\delta$ such that for all nondecreasing sequences $a_{0}, b_{0}, \ldots a_{k}, b_{k} \in E$, if $\sum_{i}\left|b_{i}-a_{i}\right|<\delta$, then $\sum_{i}\left|F\left(b_{i}\right)-F\left(a_{i}\right)\right|<$ $\varepsilon$ (respectively $\left.\sum_{i} \omega\left(F, a_{i}, b_{i}\right)<\varepsilon\right)$.

Observe that if $E$ is an interval, then being $V B$ on $E$ is the same thing as being $V B_{*}$ on $E$, and similarly for absolute continuity. We can also understand what it means for a function to satisfy these properties on a closed set $E$ with reference to simplified functions $F_{E}$ and $F_{E, *}$ defined as follows.

${ }^{1}$ Because both sets consist of machine indices for machines with access to $X \oplus Y$, the existence of a single pair of computable reductions follows from the existence of a single pair of uniformly $X \oplus Y$-computable reductions. 
Definition 3. Let $F \in C(I)$, and $E \subseteq I$ a closed set. Then let $F_{E}$ and $F_{E, *}$ denote the functions satisfying

(1) $F_{E}(x)=F_{E, *}(x)=F(x)$ for $x \in E$, and

(2) If $(c, d)$ is a connected component of $I \backslash E$,

(a) let $F_{E}$ be linear on $[c, d]$, and

(b) let

$$
\begin{aligned}
& F_{E, *}\left(\frac{2 c+d}{3}\right)=\sup F([c, d]) \\
& F_{E, *}\left(\frac{c+2 d}{3}\right)=\inf F([c, d]),
\end{aligned}
$$

and let $F_{E, *}$ be linear on $\left[c, \frac{2 c+d}{3}\right],\left[\frac{2 c+d}{3}, \frac{c+2 d}{3}\right]$ and $\left[\frac{c+2 d}{3}, d\right]$.

Note that $\omega\left(F_{E, *},[c, d]\right)=\omega(F,[c, d])$ for $(c, d)$ a connected component of $I \backslash E$.

Then the following proposition holds:

Proposition 6. Let $F \in C(I)$ and $E \subseteq I$ be closed. Then

(1) $F$ is $V B$ (resp. $A C$ ) on $E$ if and only if $F_{E}$ is $V B$ (resp. $A C$ ) on $I$.

(2) $F$ is $V B_{*}$ (resp. $A C_{*}$ ) on $E$ if and only if $F_{E, *}$ is $V B$ (resp. AC) on $I$.

Now we can define the main notions.

Definition 4. A function $F \in C(I)$ is $V B G$ (respectively $A C G, V B G_{*}, A C G_{*}$ ) if there is a countable sequence of closed sets $E_{n}$ such that $\cup_{n} E_{n}=I$ and $F$ is $V B$ (respectively $\left.A C, V B_{*}, A C_{*}\right)$ on each $E_{n}$.

It is immediate that $V B G_{*} \subseteq V B G$ and $A C G_{*} \subseteq A C G$. Recall the relationship between absolute continuity and bounded variation: a continuous function of bounded variation is absolutely continuous if and only if it satisfies Lusin's condition $(N)$.

Definition 5. A function $F: I \rightarrow \mathbb{R}$ satisfies $(N)$ if for every Lebesgue null set $A \subseteq I$, its image $F(A)$ is also null.

If $\cup_{n} E_{n}=I$, then $F$ satisfies $(N)$ if and only if it satisfies $(N)$ on each $E_{n}$. Therefore, $F \in A C G$ if and only if $F \in V B G$ and $F$ satisfies $(N)$. We also have (see [Sak64, Thm VII.8.8, pg. 233]) that $A C G_{*}=V B G_{*} \cap A C G$. It follows that $F \in A C G_{*}$ if and only if $F \in V B G_{*}$ and $F$ satisfies $(N)$.

Based on the definitions above, it would seem that these sets are $\Sigma_{2}^{1}$. However, the following equivalent characterization shows each of these classes is in fact $\Pi_{1}^{1}$.

Theorem 7 (see [Sak64, Thm VII.9.1, pg 233]). For a function $F$ to be VBG (respectively $\left.V B G_{*}, A C G, A C G_{*}\right)$ it is necessary and sufficient that for every closed $E \subseteq I$, there is an interval $[a, b] \subseteq I$ such that $(a, b) \cap E \neq \emptyset$ and $F$ is $V B$ (respectively $V B_{*}, A C, A C_{*}$ ) on $[a, b] \cap E$.

Corollary 8. The sets $V B G, V B G_{*}, A C G, A C G_{*} \subseteq C(I)$ are all $\Pi_{1}^{1}$.

The previous theorem also suggests a derivation process.

Definition 6. Let $X$ stand for $V B, V B_{*}, A C$ or $A C_{*}$. Given $F \in C(I)$, define $P_{F, X}^{0}=I$. Define

$$
P_{F, X}^{\alpha+1}=P_{F, X}^{\alpha} \backslash \cup\left\{(a, b): F \text { is } X \text { on }[a, b] \cap P_{F, X}^{\alpha}\right\}
$$


and for a limit ordinal $\lambda$, define $P_{F, X}^{\lambda}=\cap_{\alpha<\lambda} P_{F, X}^{\alpha}$. Define a rank $|\cdot|_{X}$ by letting $|F|_{X}$ be the least $\alpha$ such that $P_{F, X}^{\alpha}=\emptyset$, if such $\alpha$ exists.

If $F \in V B G$, then the only way for $P_{F, V B}^{\alpha+1}=P_{F, V B}^{\alpha}$ is if $P=\emptyset$; and furthermore, the countable sequence of sets $[a, b] \cap P_{F, V B}^{\alpha}$ for which $(a, b)$ were removed over the course of the derivations would serve as the sequence $E_{n}$ required in the definition of $V B G$. Reasoning similarly about all four hierarchies, it is immediate that a given $F \in C(I)$ belongs to one of the classes $V B G, V B G_{*}, A C G, A C G_{*}$ if and only if the associated derivation process eventually produces the empty set.

Definition 7. Let $V B G_{\alpha}$ (respectively $V B G_{* \alpha}, A C G_{\alpha}, A C G_{* \alpha}$ ) denote the sets $\{F \in C(I)$ : $\left.|F|_{V B} \leq \alpha\right\}$ (respectively $\left.|F|_{V B_{*}},|F|_{A C},|F|_{A C_{*}}\right)$.

Recall from the introduction that $A C G_{*}$ is exactly the set of functions $F \in C(I)$ which can be recovered from $F^{\prime}$ by Denjoy totalization. We will not give the definition of the Denjoy totalization process, because we almost always prefer to work with the related derivation processes. The one place where familiarity with the totalization process is needed is in the proof of Theorem 21. A definition can be found in [Sak64, Section VIII.5]. What matters for us is that Denjoy totalization is a transfinite procedure which terminates at some countable ordinal stage, and $A C G_{* \alpha}$ consists of precisely the functions $F \in A C G_{*}$ which are recovered from $F^{\prime}$ in at most $\alpha$ steps of Denjoy totalization. Therefore, the sets $A C G_{* \alpha}$ have a meaningful interpretation in terms of Denjoy totalization.

Note that there are actually two transfinite procedures which are sometimes called "Denjoy totalization": the narrow Denjoy integral, which coincides with the integrals of Perron, Kurzweil and Henstock, and the wide Denjoy integral, sometimes known as the DenjoyKhintchine integral. In this paper, "Denjoy totalization" always refers to the narrow Denjoy integral. The narrow Denjoy integral has the same relationship to the class $A C G_{*}$ as the wide Denjoy integral has to the class $A C G$.

\section{Cantor-Bendixson RAnK}

In this section we analyze the initial segments of the Cantor-Bendixson hierarchy. The theorems of this section are not used in later sections. Let $S \subseteq 2^{<\omega}$ be a tree with no dead ends. Let $[S]$ denote the set of paths in $S$. The Cantor-Bendixson derivative $D(S)$ is defined as the tree without dead ends such that $[D(S)]$ consists of exactly the paths not isolated in $[S]$. Define $D^{0}(S)=S, D^{\alpha+1}(S)=D\left(D^{\alpha}(S)\right)$, and $D^{\lambda}(S)=\cap_{\alpha<\lambda} D^{\alpha}(S)$ for $\lambda$ a limit.

Definition 8. The Cantor-Bendixson rank of a tree $S \subseteq 2^{\omega}$, denoted $|S|_{C B}$, is the least $\alpha$ such that $D^{\alpha}(S)=\emptyset$, if such exists. Otherwise we say $|S|_{C B}=\infty$.

Other authors define $|T|_{C B}$ to be the least $\alpha$ such that $D^{\alpha}(S)=D^{\alpha+1}(S)$, so that a set with a perfect subset also has a Cantor-Bendixson rank. Others define their rank to be always one less than ours, so that every ordinal is used.

Proposition 9. Let $Y \in 2^{\omega}$ and $\alpha<\omega_{1}^{Y}$. Then $\left\{S:|S|_{C B} \leq \alpha\right\}$ is $\Sigma_{2 \alpha}^{0}(Y)$ in $\{S$ : $S$ is a tree with no dead ends\}.

Proof. The proof is by effective transfinite recursion. Because checking whether a no-deadends tree is empty can be accomplished by checking the root, $\left\{S:|S|_{C B}=0\right\}$ is $\Sigma_{0}^{0}$.

A tree $S$ has $D^{\alpha+1}(S)=\emptyset$ if and only if $D^{\alpha}(S)$ has only finitely many branches. If $D^{\alpha}(T)$ has at least $k$ branches, then by going up to a height $n$ at which the branches have 
separated, we may find at least $k$-many $\sigma$ of length $n$ such that $D^{\alpha}\left(T_{\sigma}\right) \neq \emptyset$. And if there are $k$ incomparable $\sigma$ such that $D^{\alpha}\left(T_{\sigma}\right) \neq \emptyset$, then $D^{\alpha}(T)$ has at least $k$ branches. Thus

$D^{\alpha+1}(S)=\emptyset \Longleftrightarrow \exists k \forall n$ (there are at most $k$ many $\sigma$ of length $n$ for which $\left.D^{\alpha}\left(S_{\sigma}\right) \neq \emptyset\right)$.

Assuming $\left\{S: D^{\alpha}(S)=\emptyset\right\}$ is $\Sigma_{2 \alpha}^{0}(Y)$ uniformly in $\alpha$, this shows that $D^{\alpha+1}(S)=\emptyset$ is $\Sigma_{2 \alpha+2}^{0}(Y)$ uniformly in $\alpha$.

If $\lambda$ is a limit, a tree has $D^{\lambda}(S)=\emptyset$ if and only if there is an $\alpha<\lambda$ such that $D^{\alpha}(S)=\emptyset$, by compactness. Assuming $D^{\alpha}(S)=\emptyset$ is $\Sigma_{2 \alpha}^{0}(Y)$ uniformly in $\alpha<\lambda$, and supposing a sequence $\alpha_{n} \rightarrow \lambda$ is $Y$-effectively given, we have $D^{\lambda}(S)=\emptyset$ if and only if $\exists \alpha<\lambda\left[D^{\alpha}(S)=\emptyset\right]$, a $\Sigma_{\lambda}^{0}(Y)$ statement. Note $\Sigma_{\lambda}^{0}(Y)=\Sigma_{2 \lambda}^{0}(Y)$ for $\lambda$ a limit.

Proposition 10. There is a computable reduction $T \mapsto S_{T}$ from trees $T \subseteq \omega^{<\omega}$ to no-deadend trees $S \subseteq 2^{<\omega}$, satisfying

(1) If $T$ is not well-founded, $\left[S_{T}\right]$ contains a perfect set.

(2) If $T$ is well-founded with $|T|_{l s}=\alpha$, then $\left[S_{T}\right]$ is countable, and $\left|S_{T}\right|_{C B}=\alpha$.

Proof. The idea is that each node of a tree $T \subseteq \omega^{<\omega}$ should correspond to a branching of paths in $S_{T} \subseteq 2^{<\omega}$, with the topological clustering of the paths provided by the hierarchical structure of $T$.

Define $S_{T}$ by

$$
S_{T}=\left\{0^{2 n_{0}+i_{0}} 10^{2 n_{1}+i_{1}} 1 \cdots 0^{2 n_{k}+i_{k}} 10^{m}:\left(n_{0}, \ldots, n_{k}\right) \in T, i_{0}, \ldots i_{k} \in\{0,1\}\right\} .2
$$

Note that $0^{m} \in S_{T}$ if and only if the empty node is in $T$. If $T \notin W F$, then if $P \in[T]$ the following subset of $\left[S_{T}\right]$ is perfect:

$$
\left\{0^{2 P(0)+X(0)} 10^{2 P(1)+X(1)} 1 \cdots: X \in 2^{\omega}\right\} .
$$

Supposing now that $T$ is well-founded, we claim that $|T|_{l s}=\left|S_{T}\right|_{C B}$. The proof is by induction on the usual rank of $T$. If $T=\emptyset$ then also $S_{T}=\emptyset$, so $\left|S_{T}\right|_{C B}=|T|_{l s}=0$.

Suppose $|T|_{l s}=\alpha+1$. (This is the only case because the limsup rank is always a successor.) Then there is an $N$ such that for $n \geq N,\left|T_{n}\right|_{l s} \leq \alpha$. Observe that if $C \subseteq 2^{<\omega}$ is any finite prefix-free collection of strings such that $\cup_{\sigma \in C}[\sigma]$ covers $[S]$, then

$$
D^{\alpha}(S)=\bigcup_{\sigma \in C} \sigma^{\frown} D^{\alpha}\left(S_{\sigma}\right)
$$

Letting $C=\left\{0^{2 N}\right\} \cup\left\{0^{2 n+i} 1: n<N, i \in\{0,1\}\right\}$, we have

$$
D^{\alpha+1}\left(S_{T}\right)=\bigcup_{\sigma \in C} \sigma^{\wedge} D^{\alpha+1}\left(\left(S_{T}\right)_{\sigma}\right) .
$$

By induction, for $n<N$ and $i \in\{0,1\},\left|0^{2 n+i} 1^{\frown} S_{T_{n}}\right|_{C B} \leq \alpha+1$, so

$$
D^{\alpha+1}\left(S_{T}\right)=0^{2 N \wedge} D^{\alpha+1}\left(\left(S_{T}\right)_{0^{2 N}}\right) .
$$

Also, for $n \geq N$, we have $\left|0^{2 n+i} 1^{\frown} S_{T_{n}}\right|_{C B} \leq \alpha$, so for each $k, D^{\alpha}\left(\left(S_{T}\right)_{0^{2 N+k}}\right)=\emptyset$, so $\left[D^{\alpha}\left(\left(S_{T}\right)_{0^{2 N}}\right)\right] \subseteq\left\{0^{\omega}\right\}$. Therefore $D^{\alpha+1}\left(\left(S_{T}\right)_{0^{2 N}}\right)=\emptyset$, so $\left|S_{T}\right|_{C B} \leq \alpha+1$.

Now we need $\left|S_{T}\right|_{C B} \geq \alpha+1$. If $\left|T_{n}\right|_{l_{s}}=\alpha+1$ for some $n$, then by induction $\left|0^{n} 1^{\frown} S_{T_{n}}\right|_{C B}=$ $\alpha+1$, which suffices. If $\lim \sup _{n}\left|T_{n}\right|_{l s}=\alpha$, then for every $\beta<\alpha$, there are infinitely many

${ }^{2}$ The more familiar option, $S_{T}^{\prime}=\left\{0^{n_{0}} 10^{n_{1}} 1 \cdots 0^{n_{k}} 10^{m}:\left(n_{0}, \ldots, n_{k}\right) \in T\right\}$, satisfies part (2) but not part (1) of the theorem; consider the case when $[T]$ consists of a single path. 
$n$ such that $\left|T_{n}\right|>\beta$, so there are infinitely many $n$ for which $\left|0^{n} 1^{\frown} S_{T_{n}}\right|_{C B}>\beta$. Therefore, for all $\beta<\alpha, 0^{\omega}$ is not an isolated path of $D^{\beta}\left(S_{T}\right)$, so $0^{\omega} \in\left[D^{\alpha}\left(S_{T}\right)\right]$, and $\left|S_{T}\right|_{C B}>\alpha$.

We can now obtain a new proof of the results of Cenzer and Mauldin in [CM83 and CM82. Though their results are stated topologically, their reductions are computable.

Theorem 11 ([CM83, Theorem 8]). For any nonzero $\alpha<\omega_{1}$, let

$$
A_{\alpha}=\left\{S \subseteq 2^{\omega}: S \text { is a no dead end tree and }|S|_{C B} \leq \alpha\right\} .
$$

Then all the conclusions of Theorem 5 hold. In particular, if $\alpha<\omega_{1}^{C K}, A_{\alpha}$ is $\Sigma_{2 \alpha}^{0}$-complete.

Their main result, identifying the precise Borel class of the $\alpha$-derivative operator on the space of closed subsets of $2^{\omega}$, follows directly from this theorem, and they also prove this theorem on the way to their result.

A similar index set result was obtained independently by Lempp.

Corollary 12. [Lem87] For each constructive $\alpha>1$, the sets

$$
\left\{e: \phi_{e} \text { codes a tree } S \text { which has no dead ends and }|S|_{C B} \leq \alpha\right\}
$$

are $\Sigma_{2 \alpha}$-complete.

This result differs only cosmetically from the result as it was stated in in [Lem87] because he considered trees which might have dead ends and because he used a definition of $\Sigma_{2 \alpha^{-}}^{0}$ complete which is off by one from our definition for $\alpha \geq \omega$. Due to a similar difference in notational conventions, the results of [CM83] also appear to be off by one from ours in the infinite case.

\section{Completeness and hardness on the Denjoy hierarchies}

In this section we present a construction which provides a computable reduction from $(W F, \neg W F)$ to $\left(A C G_{*}, \neg V B G\right)$. Because $A C G_{*} \subseteq A C G \cap V B G_{*}$ and $A C G \cup V B G_{*} \subseteq$ $V B G$, this shows that all four classes are $\Pi_{1}^{1}$-complete. Additionally, this reduction serves as a simultaneous uniform reduction from $\left(A_{\alpha}, A_{\alpha+1}\right)$ to $\left(A C G_{* \alpha}, \neg V B G_{\alpha}\right)$, where $A_{\alpha}=\{T \in$ $\left.W F:|T|_{l s} \leq \alpha\right\}$. We have $A C G_{* \alpha} \subseteq A C G_{\alpha} \cap V B G_{* \alpha}$ and $A C G_{\alpha} \cup V B G_{* \alpha} \subseteq V B G_{\alpha}$, so all these sets are at least as complex as $A_{\alpha}$, namely at least $\Sigma_{2 \alpha}^{0}(Y)$-hard.

The idea is that each node of the given tree should contribute a finite length to the variation of the constructed function. In most cases the total variation will be infinite as a result, but the way in which that infinite length is distributed will determine the rank of the function.

The following notation will be useful: if $J, K \subseteq I$ are two intervals, then $J\langle K\rangle$ denotes the interval that has the same relation to $K$ as $J$ has to $I$; that is, if $J=[a, b]$ and $K=[c, d]$ then $J\langle K\rangle=[c+a(d-c), c+b(d-c)]$.

To define this reduction, it will be useful to have a way to tell $F_{T}$ to increase its variation in a given interval $J$ in response to seeing more of $T$. Given an interval $J \subseteq[0,1]$, we define the wiggle function $W(J)$ as follows. The goal is to have a function whose variation is at least 1 (regardless of how small $J$ is), but which only takes values in $[0,|J|]$, where $|J|$ is the length of the interval $J$. Therefore, for small $J$, the function should oscillate intensely. We also leave some space at the top and bottom of each oscillation to give room for adding some more oscillations; this sets us up for a typical method of producing a function of high Denjoy rank. 
Definition 9. Given an interval $J \subseteq I$, let $M$ be the least integer large enough that $2 M|J|>$ 1 , and define

(1) $W(J)(x)=0$ if $x \notin J$.

(2) $W(J)(x)=0$ if $x \in\left[\frac{4 k}{4 M+1}, \frac{4 k+1}{4 M+1}\right]\langle J\rangle$ for $k \leq M$.

(3) $W(J)(x)=|J|$ if $x \in\left[\frac{4 k+2}{4 M+1}, \frac{4 k+3}{4 M+1}\right]\langle J\rangle$ for $k<M$.

(4) If $x \in\left[\frac{4 k+i}{4 M+1}, \frac{4 k+i+1}{4 M+1}\right]\langle J\rangle$ for $k<M$ and $i=1,3$, determine the value of $W(J)(x)$ by linear interpolation from the values already defined.

Define

$$
\begin{aligned}
\text { Flat }(J) & =\{K \subseteq J: K \text { is a maximal interval on which } W(J) \text { is constant }\} \\
& =\left\{\left[\frac{4 k+i}{4 M+1}, \frac{4 k+i+1}{4 M+1}\right]\langle J\rangle:(k<M, i=0,2) \text { or }(k=M, i=0)\right\}
\end{aligned}
$$

Fix an infinite sequence of disjoint closed interval subsets of $I$, decreasing in size as they approach 0, with gaps between them. For specificity, say $J_{n}=\left[\frac{1}{3 n+2}, \frac{1}{3 n+1}\right]$. For any interval $L$, we define the function $\tilde{F}(T, L)$ by recursion on the usual rank of $T$ as follows.

Definition 10. Given an interval $L \subseteq I$, let $\tilde{F}(\emptyset, L)$ be the constant function 0 , and for nonempty $T$, let

$$
\tilde{F}(T, L)=W(L)+\sum_{K \in \operatorname{Flat}(L), n \in \omega} \tilde{F}\left(T_{n}, J_{n}\langle K\rangle\right) .
$$

Observe that the $\tilde{F}\left(T_{n}, J\right)$ are being copied onto the plateaus of the wiggle functions. Also, for $K, H \in \operatorname{Flat}(L)$, the functions $\tilde{F}\left(T_{n}, J_{n}\langle K\rangle\right)$ and $\tilde{F}\left(T_{m}, J_{m}\langle H\rangle\right)$ have disjoint support, unless $m=n$ and $K=H$. So for $x \notin \cup_{n, K} J_{n}\langle K\rangle$, we have $\tilde{F}(T, L)(x)=W(L)(x)$, and otherwise $\tilde{F}(T, L)(x)=W(L)(x)+\tilde{F}\left(T_{n}, J_{n}\langle K\rangle\right)(x)$ for the unique $n$ and $K$ such that $x \in J_{n}\langle K\rangle$. Furthermore, there is an open neighborhood of $J_{n}\langle K\rangle$ on which the functions $\tilde{F}(T, L)$ and $\tilde{F}\left(T_{n}, J_{n}\langle K\rangle\right)$ differ by only a constant. Therefore,

$$
P_{\tilde{F}(T, L), X}^{\alpha} \cap J_{n}\langle K\rangle=P_{\tilde{F}\left(T_{n}, J_{n}\langle K\rangle\right), X}^{\alpha} \cap J_{n}\langle K\rangle
$$

for all $\alpha$, where $X$ is any of $V B, V B_{*}, A C, A C_{*}$. This fact will be used throughout.

It is possible to extend this recursive definition of $\tilde{F}$ so that any tree $T$ (even ill-founded) can be used. Each $\sigma \in T$ contributes some wiggle to $\tilde{F}(T, L)$, in locations which can be described as follows. Let $\mathcal{C}_{\emptyset}^{L}=\{L\}$, and given $\mathcal{C}_{\sigma}^{L}$, define

$$
\mathcal{C}_{\sigma^{\urcorner} n}^{L}=\bigcup_{H \in \mathcal{C}_{\sigma}^{L}}\left\{J_{n}\langle K\rangle: K \in \operatorname{Flat}(H)\right\}
$$

Definition 11. Given an interval $L \subseteq I$, define

$$
F(T, L)=\sum_{\substack{\sigma \in T \\ H \in \mathcal{C}_{\sigma}^{L}}} W(H)
$$

Proposition 13. For all trees $T \subseteq \omega^{<\omega}, F(T, L)$ is well-defined and uniformly $T$-computable. For $T \in W F, F(T, L)=\tilde{F}(T, L)$. 
Proof. We may decompose $F(T, L)=\sum_{\ell<\omega} F_{\ell}(T, L)$, where

$$
F_{\ell}(T, L)=\sum_{\substack{\sigma \in T:|\sigma|=\ell \\ H \in \mathcal{C}_{\sigma}^{L}}} W(H),
$$

Observe that since each $J_{n}$ satisfies $\left|J_{n}\right|<1 / 2$, each $H \in \mathcal{C}_{\sigma}^{L}$ satisfies $|H| \leq 2^{-|\sigma|}$. Furthermore, the intervals of $\bigcup_{\sigma:|\sigma|=\ell} \mathcal{C}_{\sigma}^{L}$ are disjoint. Therefore, since $W(H)$ has its range in $[0,|H|]$, each $F_{\ell}(T, L)$ has its range in $\left[0,2^{-\ell}\right]$. Also, the $F_{\ell}(T, L)$ are uniformly computable. Therefore, $F(T, L)$ is the effective uniform limit of computable functions, and is therefore computable.

Now suppose $T \in W F$. If $T$ is just a root, it is clear that $F(T, L)=\tilde{F}(T, L)$. Assuming that each $F\left(T_{n}, K\right)=\sum_{\sigma \in T_{n}, H \in \mathcal{C}_{\sigma}^{K}} W(H)$, the agreement of the two definitions follows in general because for each $n$,

$$
\mathcal{C}_{n^{\urcorner} \sigma}^{L}=\bigcup_{K \in \operatorname{Flat}(L)} \mathcal{C}_{\sigma}^{J_{n}\langle K\rangle}
$$

Proposition 14. If $T$ is not well-founded, then $F(T, I) \notin V B G$.

Proof. Let $Z$ be a path in $T$, and consider

$$
P_{Z}=\bigcap_{\ell \in \omega}\left(\bigcup \mathcal{C}_{Z \uparrow \ell}^{I}\right)
$$

This is an intersection of a decreasing sequence of closed sets in a compact space, so $P_{Z}$ is closed and non-empty. We claim that there is no open interval $U$ such that $P_{Z} \cap U$ is nonempty and $F(T, I)$ has bounded variation on $P_{Z} \cap U$. By Theorem 7 , this suffices.

Fix an open interval $U$ and a number $N$. Since $P_{Z} \cap U \neq \emptyset$, there is an $M$ and $H_{0} \in \mathcal{C}_{Z\lceil M}^{I}$ such that $P_{Z} \cap H_{0} \subseteq U$. We will show that the variation of $F(T, I)$ on $P_{Z} \cap H_{0}$ is at least $2^{N}$. Let $\mathcal{C}=\left\{H \in \overline{\mathcal{C}}_{Z \uparrow(M+N)}^{I}: H \subseteq H_{0}\right\}$. Since each interval of $\mathcal{C}_{Z\lceil\ell}^{I}$ is broken into multiple intervals in $\mathcal{C}_{Z \uparrow(\ell+1)}^{I}$, there are at least $2^{N}$ intervals in $\mathcal{C}$.

Let the functions $F_{\ell}$ be as in the previous proposition. For each $H \in \mathcal{C}, \sum_{\ell<M+N} F_{\ell}(T, I)$ is constant on $H$, the function $W(H)$ has variation at least 1 , and $F_{M+N}(T, I)(x)=W(H)(x)$ for all $x \in H$. Therefore, the function $G$ defined by $G=\sum_{\ell \leq M+N} F_{\ell}(T, I)$ has variation at least 1 on $H$. In fact, $G$ has variation at least 1 on any subset of $H$ which contains at least one point of $K$ for each $K \in \operatorname{Flat}(H)$. Therefore, it suffices to show that each such $K$ contains a point $x \in P_{Z}$ such that $G(x)=F(T, I)(x)$.

We claim that $x=\min \left(P_{Z} \cap K\right)$ is such a point. For each $\ell>M+N$, there is a unique interval $J \in \cup_{\sigma \in T:|\sigma|=\ell} \mathcal{C}_{\sigma}^{I}$ such that $x \in J$. All those intervals are disjoint, so $F_{\ell}(T, I)(x)=$ $W(J)(x)$. We claim that $W(J)(x)=0$. Since $x \in P_{Z}, J \in \mathcal{C}_{Z\lceil\ell}^{I}$. Because $x$ is the minimum of $P_{Z} \cap K$, it is also the minimum of $P_{Z} \cap J$. But $P_{Z}$ contains points from every $L \in$ Flat $(J)$. Therefore, $x$ is an element of the first such interval $L \subseteq J$. Since $W(J) \equiv 0$ on its first interval, $W(J)(x)=0$, as required. Since this is true for arbitrary $\ell$, we have $G(x)=F(T, I)(x)$ for all such $x$. Therefore, the variation of $F(T, I)$ on $H_{0}$ is at least $2^{N}$, so $F(T, I) \notin V B G$.

Proposition 15. If $T$ is well-founded and $|T|_{l s} \geq 1$, then $F(T, I) \in A C G_{*}$, and $|F(T, I)|_{X}=$ $|T|_{l s}$, where $X$ is any of $V B, V B_{*}, A C, A C_{*}$. 
Proof. We proceed by induction on the usual rank of the tree, starting with the root-only tree $T=\{\emptyset\}$. The statement we prove inductively is slightly stronger: for all intervals $L$, if $|T|_{l s}=\alpha+1$, then

- $|F(T, L)|_{X}=\alpha+1$, and

- for each $K \in \operatorname{Flat}(L)$, there is a point $x_{K} \in K$ such that $x_{K} \in P_{F(T, L), X}^{\alpha}$ and $F(T, L)(x)=W(L)(x)$.

From here forward, we drop the subscripts on $P_{F(T, L), X}^{\alpha}$, whenever possible, always omitting $X$, and writing $P^{\alpha}$ instead of $P_{F(T, L)}^{\alpha}$. When subscripts are included, it is because we reference the derivation applied to a different function, typically $F\left(T_{n}, J_{n}\langle K\rangle\right)$ for some $n$ and some $K$.

In the base case, the tree $T=\{\emptyset\}$ has $|T|_{l s}=1$, and $F(T, L)=W(L)$, so it also has rank 1 , and the existence of appropriate $x_{K}$ is immediate. Let $T$ be given, with $|T|_{l s}=\alpha+1$. If $\alpha=0$, then just as in the base case, $P^{0}=L$, so letting $x_{K}=\min K$ for each $K \in \operatorname{Flat}(L)$ suffices. So assume that $\alpha \geq 1$. By induction, for each $n,\left|T_{n}\right|_{l s}=\left|F\left(T_{n}, J_{n}\langle K\rangle\right)\right|_{X}$. We know that

$$
P^{\alpha} \subseteq \bigcup_{K \in \operatorname{Flat}(L)}\{\min K\} \cup \bigcup_{n} J_{n}\langle K\rangle,
$$

because $F(T, L)$ is absolutely continuous outside this set. By the discussion following Definition 10, because $\left|T_{n}\right|_{l s} \leq \alpha+1$ for all $n$, we have $P^{\alpha+1} \cap J_{n}\langle K\rangle=\emptyset$ for all $n$ and $K$. Similarly, because $\left|T_{n}\right|_{l s} \leq \alpha$ for sufficiently large $n$, we also have $P^{\alpha} \cap J_{n}\langle K\rangle=\emptyset$ for sufficiently large $n$. Therefore, if $\min K \in P^{\alpha}$, it is isolated, so for all $K$, we have $\min K \notin P^{\alpha+1}$. This shows that $|F(T, L)|_{X} \leq \alpha+1$.

On the other hand, suppose that $|T|_{l s}=\alpha+1$. Fix $K \in \operatorname{Flat}(L)$. Observe that finding a point $x_{K}$ as above suffices to complete the argument, because $x_{K}$ witnesses that $P^{\alpha} \neq \emptyset$, and so $|F(T, L)|_{X} \geq \alpha+1$. We consider three cases, which are not mutually exclusive, but which exhaust all possibilities.

Case 1. Suppose $\left|T_{n}\right|_{l s}=\alpha+1$ for some $n$. Then, letting $H \in \operatorname{Flat}\left(J_{n}\langle K\rangle\right)$ be leftmost, by induction let $x_{K} \in H$ with $x_{K} \in P_{F\left(T_{n}, J_{n}\langle K\rangle\right)}^{\alpha}$ and $F\left(T_{n}, J_{n}\langle K\rangle\right)\left(x_{K}\right)=W\left(J_{n}\langle K\rangle\right)\left(x_{K}\right)=0$, where the last equality follows because $H$ was chosen leftmost. Observe that $x_{K} \in P^{\alpha}$ as well, and $F(T, L)\left(x_{K}\right)=W(L)\left(x_{K}\right)+F\left(T_{n}, J_{n}\langle K\rangle\right)\left(x_{K}\right)=W(L)\left(x_{K}\right)$.

Case 2. Suppose that $\alpha$ is a limit and $\alpha=\lim \sup _{n}\left|T_{n}\right|_{l s}$. In this case we may let $x_{K}=\min K$. It is immediate that $F(T, L)\left(x_{K}\right)=W(L)\left(x_{K}\right)$, because no other summand in Definition 10 has $x_{K}$ in its support. For any $\beta<\alpha$, there are infinitely many $n$ such that $\left|T_{n}\right|_{l s}>\beta$. For each such $n$, by induction there is some $x_{n} \in J_{n}\langle K\rangle$ such that $x_{n} \in$ $P_{F\left(T_{n}, J_{n}\langle K\rangle\right)}^{\beta}$. We also have $x_{n} \in P^{\beta}$ for all such $n$. Since $x_{K}$ is the limit of of this collection of $x_{n}$, it follows that $x_{K} \in P^{\beta}$, because $P^{\beta}$ is closed. This was done for an arbitrary $\beta<\alpha$, so $x_{K} \in P^{\alpha}$.

Case 3. Suppose that $\alpha=\beta+1$ and $\alpha=\lim \sup _{n}\left|T_{n}\right|_{l s}$. Then for infinitely many $n$, we have $\left|T_{n}\right|_{l s}=\alpha$. Let $x_{K}=\min K$. As above, we have $F(T, L)\left(x_{K}\right)=W(L)\left(x_{K}\right)$, so it remains only to show that $x_{K} \in P^{\alpha}$.

For each $n$ such that $\left|T_{n}\right|_{l s}=\alpha$, and each $H \in \operatorname{Flat}\left(J_{n}\langle K\rangle\right)$, by induction let $x_{n, H} \in H$ be such that $x_{n, H} \in P_{F\left(T_{n}, J_{n}\langle K\rangle\right)}^{\beta}$ and

$$
F\left(T_{n}, J_{n}\langle K\rangle\right)\left(x_{n, H}\right)=W\left(J_{n}\langle K\rangle\right)\left(x_{n, H}\right) .
$$


We also have each such $x_{n, H} \in P^{\beta}$. Now observe that the variation of $W\left(J_{n}\langle K\rangle\right)$ on $P^{\beta}$ is at least 1, because $P^{\beta}$ contains one point $x_{n, H}$ in each interval $H \in \operatorname{Flat}\left(J_{n}\langle K\rangle\right)$. By (2), the variation of $F\left(T_{n}, J_{n}\langle K\rangle\right)$ is at least 1 on $P^{\beta}$, so in view of the discussion following Definition 10, the variation of $F(T, L)$ is also at least 1 on $P^{\beta} \cap J_{n}\langle K\rangle$. In any neighborhood of $x_{K}$, there are infinitely many intervals $J_{n}\langle K\rangle$ for which this is true. Therefore, $F(T, L)$ is not $V B$ on $P^{\beta}$ in any neighborhood of $x_{K}$. This shows that $x_{K} \in P^{\alpha}$.

Corollary 16. Let $Y \in 2^{\omega}$ and $\alpha<\omega_{1}^{Y}$. For any $\Sigma_{2 \alpha}^{0}(Y)$ set $B$, there is a $Y$-computable reduction from $(B, \neg B)$ to $\left(A C G_{* \alpha}, \neg V B G_{\alpha}\right)$.

Theorem 17. The sets $V B G, V B G_{*}, A C G, A C G_{*}$ are all $\Pi_{1}^{1}$-complete.

This answers a question of Walsh [Wal17], who asked whether $A C G_{*}$ was $\Pi_{1}^{1}$-complete. Walsh also showed that the graph of Denjoy integration is a $\Pi_{1}^{1}$, non-Borel subset of $M(I) \times$ $C(I)$. He asked whether that set is $\Pi_{1}^{1}$-complete, a question which we answer in the affirmative. From here on, let $F_{T}=F(T, I)$.

Lemma 18. For all trees $T \subseteq \omega^{<\omega}, F_{T}$ is a.e differentiable, and the map $T \mapsto\left(F_{T}^{\prime}, F_{T}\right) \in$ $M(I) \times C(I)$ is computable.

Proof. For each $\ell$, let

$$
G_{\ell}=\sum_{\substack{\sigma \in T:|\sigma|<\ell \\ \text { and } \max (\sigma)<\ell \\ H \in \mathcal{C}_{\sigma}^{I}}} W(H) .
$$

Then $\lim _{\ell \rightarrow \infty} G_{\ell}=F_{T}$. Observe that $G_{\ell}^{\prime}$ is a.e. equivalent to an ideal point of $M(I)$. We claim that in $M(I), \lim _{\ell \rightarrow \infty} G_{\ell}^{\prime}=F_{T}^{\prime}$. For this it suffices to observe that $G_{\ell}=F_{T}$ on any interval where $G_{\ell}^{\prime} \neq 0$, and also on any interval that is disjoint from all intervals of $\mathcal{C}_{\sigma}^{I}$ for $\sigma \in T$ with $|\sigma| \geq \ell$ or $\max (\sigma) \geq \ell$. Regardless of whether $T$ is well-founded, the measure of these intervals of agreement approaches 1 in the limit, and the convergence is effective.

Theorem 19. The set $\left\{(f, F) \in M(I) \times C(I): F \in A C G_{*}\right.$ and $F^{\prime}=f$ a.e. $\}$ is $\Pi_{1}^{1}$-complete. Proof. The map $T \mapsto\left(F_{T}^{\prime}, F_{T}\right)$ provides a computable reduction from $W F$ to $\{(f, F) \in$ $M(I) \times C(I): F \in A C G_{*}$ and $F^{\prime}=f$ a.e. $\}$.

The next and final result of this section concerns $\{f \in M(I): f$ is Denjoy integrable $\}$. Walsh showed that this set is $\boldsymbol{\Sigma}_{2}^{1}$ and not $\boldsymbol{\Sigma}_{1}^{1}$, and asked for better bounds, which we give in Theorem 22. We need a lemma.

Lemma 20. If $T \notin W F$, then $F_{T}^{\prime}$ is not Denjoy integrable.

Proof. Let $P=\cap_{\ell \in \omega} \overline{\cup D_{\ell}}$, where

$$
D_{\ell}=\left\{H: H \in \mathcal{C}_{\sigma}^{I}, \sigma \in T,|\sigma|=\ell \text { and } T_{\sigma} \notin W F\right\}
$$

Then $F_{T}$ is not $V B$ on $P \cap U$ for any open interval $U$ such that $P \cap U \neq \emptyset$, by a modification of the argument in Proposition 14. Let $H_{0} \subseteq U$ with $H_{0} \in D_{M}$ for some $M$, and let $H \subseteq H_{0}$ with $H \in D_{M+N}$. There are at least $2^{N}$ such $H$, all disjoint. Then as before, the variation of $F_{T}$ on each such $H$ is at least 1. Furthermore, if Flat $(H)=\left\{K_{0}, K_{1}, \ldots, K_{r}\right\}$, listed from left to right, then this variation is witnessed by the intervals $\left(\max \left(K_{i} \cap P\right), \min \left(K_{i+1} \cap P\right)\right)$ for each $i<r$, and these intervals are disjoint from $P$.

We claim that the restriction of $F_{T}$ to any closed interval $J$ disjoint from $P$ is $A C G_{*}$. Such an interval is disjoint from $\cup D_{\ell}$ for some $\ell$, and therefore $F_{T} \uparrow J=F_{S} \uparrow J$, where $S$ 
is the well-founded tree obtained from $T$ by removing all $\sigma$ of length at least $\ell$ such that $T_{\sigma\lceil\ell} \notin W F$. Because $F_{S}$ is $A C G_{*}, F_{T} \uparrow J$ is $A C G_{*}$.

Now suppose, for the sake of contradiction, that there is $G \in A C G_{*}$ with $G^{\prime}=F_{T}^{\prime}$. Then $G$ would be $V B$ on some portion of $P$. But for each connected component $(c, d)$ of $I \backslash P$, we have $G(d)-G(c)=F_{T}(d)-F_{T}(c)$ (because, using the continuity of $F_{T}$ and $G$, both are equal to the limit as $J$ expands to $(c, d)$ of the Denjoy integral of $F_{T}^{\prime} \uparrow J$ ), and $F_{T}$ is not $V B$ on any portion of $P$, via a sequence that uses such intervals $(c, d)$ as witnesses. Such a sequence also witnesses that $G$ has variation at least $2^{N}$ on $P \cap U$.

We also need a slight variation on the result, originally due to Ajtai, that for sequences $\bar{f} \in C(I)^{\omega}$, if $\bar{f}$ converges to $f$ and $f$ is the derivative of an everywhere differentiable function $F$, then $F \in \Delta_{1}^{1}(\bar{f})$.

Theorem 21 (essentially Ajtai, unpublished). If $f \in M(I)$ and $F \in A C G_{*}$ with $F^{\prime}=f$ a.e., then $F \in \Delta_{1}^{1}(f)$.

Proof. We refer the reader to Dougherty and Kechris [DK91, pg. 162] for the proof of Ajtai's theorem; we only need to check that the proof still works if the function $f$ is given as a measurable function (i.e as a Cauchy sequence in the metric space $M(I)$ ) that is Denjoy integrable, rather than as an element of $C(I)^{\omega}$ which must be the derivative of an everywhere differentiable function.

For the first modification, it suffices to verify that it is uniformly $\Delta_{1}^{1}$ to determine whether a given function $f \in M(I)$ is Lebesgue integrable, and if so, what is $\int f$. Recall that a non-negative function $f \in M(I)$ is Lebesgue integrable if and only if the sequence $\left\langle f_{n}\right\rangle_{n \in \omega}$ is Cauchy in the $\|\cdot\|_{1}$ norm, where $f_{n}(x)=\min (n, f(x))$. The operation $\|\cdot\|_{1}$ is computable on bounded measurable functions when the bound is also given as an input. Therefore, it is uniformly $\Delta_{1}^{1}$ to determine whether an arbitrary given $f$ is integrable, by applying the above criterion to $|f|$. If $f$ is integrable, we may calculate $\int f=\lim _{n} \int f^{n}$, where $f^{n}(x)=\max (-n, \min (n, f(x)))$. Therefore, all the operations needed to carry out the Denjoy totalization process on $f$ are uniformly $\Delta_{1}^{1}$.

The second difference in the theorem statement is that we only assume $F \in A C G_{*}$, rather than $F$ being everywhere differentiable. However, the only place it was used that $F$ is everywhere differentiable (aside from making $f$ everywhere defined in its representation as a Baire 1 function) is the assumption that the totalization process does terminate.

Theorem 22. The set $\{f \in M(I): f$ is Denjoy integrable $\}$ is $\Pi_{1}^{1}$-complete.

Proof. By Theorem 21, for $f \in M(I)$,

$f$ is Denjoy integrable $\Longleftrightarrow \exists F \in \Delta_{1}^{1}(f)\left[F \in A C G_{*}\right.$ and $F^{\prime}=f$ a.e. $]$

which is $\Pi_{1}^{1}$.

Now consider the completeness direction. By Lemma 18, the map $T \mapsto F_{T}^{\prime}$ is computable, and $T \in W F$ if and only if $F_{T}^{\prime}$ is Denjoy integrable by Lemma 20.

\section{Descriptive Results on the Denjoy hierarchies}

4.1. Descriptive complexity of $V B G$ and $V B G_{*}$ hierarchies. Our goal now is to give the precise descriptive complexity of the sets $V B G_{* \alpha}, V B G_{\alpha}$ and $A C G_{* \alpha}$. For $A C G_{\alpha}$, we will only give an upper bound on the descriptive complexity.

Proposition 23. If $\alpha<\omega_{1}^{Y}$, then $V B G_{* \alpha}$ and $V B G_{\alpha}$ are $\Sigma_{2 \alpha}(Y)$. 
Proof. By effective transfinite recursion. Let $X$ be one of $V B, V B_{*}$, and let $p, q \in \mathbb{Q}$. When $\alpha=1$, we have $P_{F, X}^{1} \cap(p, q)=\emptyset$ if and only if $F$ has bounded variation on the interval $[p, q]$. This property is $\Sigma_{2}^{0}$ uniformly in $F, p$ and $q$.

Supposing that $\left\{(F, p, q):[p, q] \cap P_{F, X}^{\alpha}=\emptyset\right\}$ is $\Sigma_{2 \alpha}^{0}(Y)$ uniformly in $\alpha$, let us show $\{(F, p, q)$ : $\left.[p, q] \cap P_{F, X}^{\alpha+1}=\emptyset\right\}$ is $\Sigma_{2 \alpha+2}(Y)$ uniformly in $\alpha$. We have by Proposition 6 ,

$$
\begin{aligned}
P_{F, V B}^{\alpha+1} \cap[p, q] & =\emptyset \Longleftrightarrow \exists\left[p^{\prime}, q^{\prime}\right] \supsetneq[p, q] \text { such that } F_{P^{\alpha}} \text { is } V B \text { on }\left[p^{\prime}, q^{\prime}\right] \\
P_{F, V B_{*}}^{\alpha+1} \cap[p, q] & =\emptyset \Longleftrightarrow \exists\left[p^{\prime}, q^{\prime}\right] \supsetneq[p, q] \text { such that } F_{E, *} \text { is } V B \text { on }\left[p^{\prime}, q^{\prime}\right] \text {, where } E=P^{\alpha} .
\end{aligned}
$$

So it is sufficient to show that there is a uniform procedure for computing, given $\varepsilon$, approximations to $F_{P^{\alpha}}$ and $F_{P^{\alpha}, *}$ that are correct to within $\varepsilon$, using an oracle which can answer $\Sigma_{2 \alpha}^{0}(F, Y)$ questions, for example questions of the form " $P_{F, X}^{\alpha} \cap[a, b]=\emptyset$ ?"

Using $F$ and the compactness of $I$, compute a number $N$ large enough that

$$
\omega\left(F,\left[k / 2^{N},(k+1) / 2^{N}\right]\right)<\varepsilon
$$

for all $k<2^{N}$. For each $\ell<k \leq 2^{N}$, ask whether $P_{F, X}^{\alpha} \cap\left[\ell / 2^{N}, k / 2^{N}\right]=\emptyset$. This identifies (up to an error of $1 / 2^{N}$ on each side) the connected components of $I \backslash P_{F, X}^{\alpha}$. Let $E$ be the corresponding approximation to $P_{F, X}^{\alpha}$; that is,

$$
E=I \backslash \cup\left\{\left(\ell / 2^{N}, k / 2^{N}\right): P_{F, X}^{\alpha} \cap\left[\ell / 2^{N}, k / 2^{N}\right]=\emptyset\right\} .
$$

Let $F^{\varepsilon}$ be an approximation of $F$ correct to within $\varepsilon$. Then return $F_{E}^{\varepsilon}$ or $F_{E, *}^{\varepsilon}$ as appropriate. The returned function will be correct to within $\varepsilon$ on $E$; on components of $I \backslash E$ that were too small to find, $F_{E, *}^{\varepsilon}$ could be off by at most $4 \varepsilon$; on components of $I \backslash E$ whose endpoints were only approximated, $F_{E, *}^{\varepsilon}$ could be off by at most $5 \varepsilon$. The errors for $F_{E}^{\varepsilon}$ are less.

Therefore, by induction, $\left\{(F, p, q): P_{F, X}^{\alpha+1} \cap[p, q]=\emptyset\right\}$ is $\Sigma_{2 \alpha+2}^{0}(Y)$, uniformly in $\alpha$.

Finally, if a limit ordinal $\lambda$ is given as a $Y$-effective sequence $\alpha_{n}$ with $\lim _{n \rightarrow \infty} \alpha_{n}=\lambda$, then $[p, q] \cap P_{F, X}^{\lambda}=\emptyset \Longleftrightarrow \exists n[p, q] \cap P^{\alpha_{n}}=\emptyset$. Since the statements $[p, q] \cap P_{F, X}^{\alpha_{n}}$ are uniformly $\Sigma_{2 \alpha_{n}}^{0}(Y)$, we have $\left\{(F, p, q):[p, q] \cap P_{F, X}^{\lambda}=\emptyset\right\}$ is $\Sigma_{\lambda}^{0}(Y)=\Sigma_{2 \lambda}^{0}(Y)$, uniformly in $\lambda$.

Observe that in the proof of Proposition 23, the only place it is used that $X$ is $V B$ or $V B_{*}$, rather than $A C$ or $A C_{*}$, is in counting the quantifiers of bounded variation in the successor step, to conclude that two jumps on an oracle for " $P_{F, X}^{\alpha} \cap[a, b]=\emptyset$ ?" questions would suffice to answer $P_{F, X}^{\alpha+1} \cap[a, b]=\emptyset$ questions. If $X$ were $A C$ or $A C_{*}$, this argument gives a bound of 4 jumps. In the case of $A C_{*}$, in the next section we will improve the bound to 2 jumps.

Corollary 24. If $\alpha<\omega_{1}^{Y}, A C G_{* \alpha}$ and $A C G_{\alpha}$ are $\Sigma_{4 \alpha}^{0}(Y)$.

4.2. Descriptive complexity of the $A C G_{*}$ hierarchy. Next we consider the descriptive complexity of $A C G_{*}$ hierarchy. Already at the first level there is a difference, because $P_{F, A C_{*}}^{1}=\emptyset$ if and only if $F$ is absolutely continuous, a $\Pi_{3}^{0}$ property.

Theorem 25. The set $\{F \in C(I): F$ is absolutely continuous $\}$ is $\Pi_{3}^{0}$-complete.

Proof. Our strategy is to define a computable reduction from $2^{\omega}$ to $C(I, I)$ whose output approximates a version of the Cantor function (Devil's Staircase function) which will converge to a Cantor-like function only if the input is an element of a given $\Pi_{3}^{0}$-complete subset of $2^{\omega}$. Let $A \subseteq 2^{\omega}$ be such a set and let $g(n)$ be a computable function such that for all $X$,

$$
X \in A \Longleftrightarrow \forall n\left[W_{g(n)}^{X} \text { is finite }\right]
$$


We now define a function $F:[0,1] \rightarrow[0,1]$, uniformly in $X$, such that $F$ is absolutely continuous if and only if $\forall n\left[W_{g(n)}^{X}\right.$ is finite $]$ holds.

Effective in $X$, we define a computable sequence of functions $F_{s}$ which converge effectively and uniformly to the desired computable function $F$. Let $F_{0}(x)=x$. Each $F_{s}$ will be piecewise linear, containing some pieces of slope zero separated by pieces of positive slope. Wherever $F_{s}$ is piecewise constant, it is equal to the limiting function $F$.

For each $n$ let $I_{n}=\left[\frac{1}{n+2}, \frac{1}{n+1}\right]$. This is the interval in which $W_{g(n)}^{X}$ 's finiteness or lack thereof will be expressed. At stage $s+1$, let $F_{s+1}\left\lceil\left[\frac{1}{n+2}, \frac{1}{n+1}\right]=F_{s}\left\lceil\left[\frac{1}{n+2}, \frac{1}{n+1}\right]\right.\right.$ for all $n \geq s$ and for all $n<s$ such that no new element of $W_{g(n)}^{X}$ has been enumerated at stage $s$. For those $n$ for which a new element is enumerated into $W_{g(n)}^{X}$, define $F_{s+1}\left\lceil I_{n}\right.$ as follows. For each maximal interval $I \subseteq I_{n}$ on which $F_{s}$ is constant, let $F_{s+1} \equiv F_{s}$ on $I$. For each maximal interval $I$ on which $F_{s}$ is linear with positive slope, define $F_{s+1}$ on $I$ to satisfy:

(1) $F_{s+1}=F_{s}$ at the endpoints of $I$

(2) $F_{s+1}$ is piecewise linear, continuous, and increasing

(3) $F_{s+1}$ has slope zero on $\frac{1}{3}$ of the measure of $I$, and positive slope everywhere else on I.

(4) $F_{s}$ and $F_{s+1}$ differ by no more than $2^{-s}$ at any point.

This can be accomplished by letting $F_{s+1}\lceil I$ resemble a sufficiently fine staircase. The effect is that $\frac{1}{3}$ of the measure of $I$ is given to points at which $F^{\prime}(x)=0$. Thus if $F_{s}^{\prime}$ was nonzero on a measure $r$ subset of $I_{n}$, then $F_{s+1}^{\prime}$ is nonzero on a measure $\frac{2}{3} r$ subset of $I_{n}$.

This completes the construction. One may check that $F$ is continuous and of bounded variation.

Now suppose that it holds that $\forall n\left[W_{g(n)}^{X}\right.$ is finite $]$. Then for each $n$, there will come a stage $s$ for which $F_{s}\left\lceil I_{n}=F\left\lceil I_{n}\right.\right.$, and so the final $F$ is piecewise linear on $I_{n}$ for all $n$. Then $F$ satisfies the Lusin $(N)$ property because each $I_{n}$ satisfies it, and there are only countably many $I_{n}$. Thus $F$ is absolutely continuous.

On the other had, suppose that $W_{g(n)}^{X}$ is infinite for some fixed $n$. Then letting $Z=$ $\cup_{s}\left\{x \in I_{n}: F_{s}^{\prime}(x)=0\right\}$, we have $\mu(Z)=\mu\left(I_{n}\right)$, but $F(Z)$ is countable, since for each $s$, $\left\{F_{s}(x): F_{s}^{\prime}(x)=0\right\}$ is finite. But $F$ is continuous, so $F\left(I_{n}\right)=I_{n}$, so $F\left(I_{n} \backslash Z\right)$ has measure $\mu\left(I_{n}\right)$, and $F$ fails to satisfy Lusin's $(N)$.

Recall that $F \in A C G$ (respectively $A C G_{*}$ ) if and only if $F$ is in $V B G$ (respectively $V B G_{*}$ ) and $F$ satisfies $(N)$. However, the set of continuous functions satisfying $(N)$ is $\Pi_{1}^{1}$-complete in the set of continuous functions [HPZvcZ99], so for the purposes of this analysis, it is easier to use a related and strictly stronger condition, Banach's condition $(S)$.

Definition 12. A function $F: I \rightarrow \mathbb{R}$ satisfies $(S)$ if for every $\varepsilon$ there is a $\delta$ such that for every set $A \subseteq I$ of Lebesgue measure less than $\delta$, its image $F(A)$ has measure less than $\varepsilon$.

In case $F$ is continuous, condition $(S)$ has a $\Pi_{3}^{0}$ equivalent definition similar to the definition of absolute continuity. For the rest of this section we adopt the convention that if $b<a$, $[a, b]$ will denote the interval $[b, a]$.

Definition 13. A function $F: I \rightarrow \mathbb{R}$ satisfies interval- $(S)$ if for every $\varepsilon$ there is a $\delta$ such that for all non-decreasing sequences $a_{0}, b_{0}, \ldots a_{k}, b_{k}$, if $\sum_{i}\left(b_{i}-a_{i}\right)<\delta$ and the intervals $\left[F\left(a_{i}\right), F\left(b_{i}\right)\right]$ are disjoint, then $\sum_{i}\left|F\left(b_{i}\right)-F\left(a_{i}\right)\right| \leq \varepsilon$.

Proposition 26. A function $F \in C(I)$ satisfies $(S)$ if and only if it satisfies interval-(S). 
Proof. If $F$ satisfies $(S)$, then by the continuity of $F$, we have that $\left[F\left(a_{i}\right), F\left(b_{i}\right)\right] \subseteq F\left(\left[a_{i}, b_{i}\right]\right)$. Therefore, $F$ satisfies interval- $(S)$ with the same witnesses that it uses to satisfy $(S)$. On the other hand, suppose $F$ satisfies interval- $(S)$, and let $\varepsilon$ be given. Let $\varepsilon_{0}<\varepsilon$. Let $\delta$ be the witness that $F$ satisfies $(S)$ for $\varepsilon_{0}$, and let $A \subseteq I$ with $\mu(A)<\delta$. Then $A$ can be covered by $\cup_{i<\omega}\left(a_{i}, b_{i}\right)$ where $\mu\left(\cup_{i<\omega}\left(a_{i}, b_{i}\right)\right)<\delta$ as well. It suffices to show that for each $k$ and for each $\varepsilon^{\prime}>0$, that $\mu\left(B_{k}\right)<\varepsilon_{0}+\varepsilon^{\prime}$, where $B_{k}=\cup_{i<k} F\left(\left[a_{i}, b_{i}\right]\right)$. By continuity of $F, B_{k}$ is a finite union of intervals. Let $\left(c_{j}, d_{j}\right)_{j<\ell}$ be chosen so that

- For each $j$, there is an $i$ such that $\left[c_{j}, d_{j}\right] \subseteq F\left(\left[a_{i}, b_{i}\right]\right)$,

- The $\left[c_{j}, d_{j}\right]$ are disjoint, and

- $\mu\left(B_{k} \backslash \cup_{j<\ell}\left[c_{j}, d_{j}\right]\right)<\varepsilon^{\prime}$.

For each $j$, choose $a_{j}^{\prime}$ and $b_{j}^{\prime}$ so that for some $i, a_{j}^{\prime}, b_{j}^{\prime} \in\left[a_{i}, b_{i}\right], F\left(\left[a_{j}^{\prime}, b_{j}^{\prime}\right]\right)=\left[c_{j}, d_{j}\right]$, and $F\left(\left\{a_{j}^{\prime}, b_{j}^{\prime}\right\}\right)=\left\{c_{j}, d_{j}\right\}$. Then the $\left(a_{j}^{\prime}, b_{j}^{\prime}\right)_{i<\ell}$ are set up as in the definition of interval- $(S)$, so $\mu\left(\cup_{j<\ell}\left[c_{j}, d_{j}\right]\right) \leq \varepsilon$, so $\mu\left(B_{k}\right)<\varepsilon+\varepsilon^{\prime}$.

Proposition 27. If $F$ is $A C G_{*}$, then $F$ fulfills condition $(S)$.

Proof. We make use of Banach's property $\left(T_{1}\right)$. By definition, a function $F$ satisfies Banach's property $\left(T_{1}\right)$ if $\left\{x \in \mathbb{R}: F^{-1}(\{x\})\right.$ is infinite $\}$ is null. It is known (cf. [Sak64, Thm IX.8.4, pg 284; Thm IX.6.3, pg 279]) that a continuous function satisfies $(S)$ if and only if it satisfies $(N)$ and $\left(T_{1}\right)$, and that any $F \in V B G_{*}$ satisfies $\left(T_{1}\right)$. Since any $F \in A C G$ satisfies $(N)$, it follows that any $F \in A C G_{*}$ satisfies $(N)$ and $\left(T_{1}\right)$, and therefore $(S)$.

Proposition 28. Let $\alpha<\omega_{1}^{Y}$ and $\alpha>1$. Then $A C G_{* \alpha}$ is $\Sigma_{2 \alpha}^{0}(Y)$.

Proof. Recall that a continuous function $F$ is absolutely continuous if and only if it is of bounded variation and satisfies property $(N)$. Furthermore, for any closed set $E$, the functions $F_{E}$ and $F_{E, *}$ have property $(N)$ if $F$ does, because their linear portions cannot contribute to a failure of $(N)$. Therefore, if $F$ has the property $(N)$, then we have not only that $F \in A C G$ (resp. $A C G_{*}$ ) if and only if $F \in V B G$ (resp. $V B G_{*}$ ), but also that $|F|_{A C}=|F|_{V B}$ (resp. $|F|_{A C_{*}}=|F|_{V B_{*}}$ ) in this case. This is because in the derivation process, the functions $F_{E}$ (respectively $F_{E, *}$ ) are absolutely continuous on an interval if and only if they are of bounded variation on that interval. Since $(N)$ is also necessary for members of $A C G$ and $A C G_{*}$, we have

$$
A C G_{\alpha}=V B G_{\alpha} \cap\{F \in C(I): F \text { satisfies }(N)\}
$$

and similarly for $A C G_{* \alpha}$. However, due to the $\Pi_{1}^{1}$-complete complexity [HPZvcZ99] of determining whether $F$ satisfies $(N)$, we cannot use this. What we can use in the case of $A C G_{*}$ is the $\Pi_{3}^{0}$ property interval- $(S)$, which is implied by $A C G_{*}$ and implies $(N)$. Therefore,

$$
A C G_{* \alpha}=V B G_{* \alpha} \cap\{F \in C(I): F \text { satisfies interval- }(S)\}
$$

and a similar statement does NOT hold for $A C G$, as there are functions in $A C G$ that do not satisfy $(S)$. Since satisfying interval- $(S)$ is only $\Pi_{3}^{0}$, this equality and Proposition 23 together complete the proof for all $\alpha>1$.

We conclude this section by summarizing the results into the main theorem mentioned in the introduction.

Theorem 29. Let $Y \in 2^{\omega}$, let $1<\alpha<\omega_{1}^{Y}$, and let $A_{\alpha}=V B G_{* \alpha}, V B G_{\alpha}$ or $A C G_{* \alpha}$. Then $A_{\alpha}$ is $\Sigma_{2 \alpha}^{0}(Y)$, and for any $\Sigma_{2 \alpha}^{0}(Y)$ set $B$, there is a $Y$-computable reduction from $B$ to $A_{\alpha}$. In particular, $A_{\alpha}$ is $\Sigma_{2 \alpha}^{0}$-complete, and if $\alpha<\omega_{1}^{C K}$, then $A_{\alpha}$ is $\Sigma_{2 \alpha}^{0}$-complete. 


\section{Questions}

Letting $|T|$ denote the usual well-founded rank of a tree $T \in W F$, a proof of the following in slightly different language can be found in [GMS13, Propositions 2.12 \& 2.15].

Theorem 30 ([GMS13]). If $\alpha<\omega_{1}^{c k}$, then $\{T:|T| \leq \omega \alpha\}$ is $\Sigma_{2 \alpha}^{0}$-complete.

Therefore, the complexity of initial segments of the usual well-founded rank increases by two jumps for every increase of $\omega$ in the rank. By contrast, all of the natural ranks considered in this paper have an increased complexity of two jumps for every increase of 1 in the rank. We wonder if there are examples of natural $\Pi_{1}^{1}$ ranks that don't fit one of these two patterns.

Question 31. Is there an example of a $\Pi_{1}^{1}$ set $A$, a natural $\Pi_{1}^{1}$ rank which decomposes it as $A=\cup_{\alpha<\omega_{1}} A_{\alpha}$, and ordinals $\beta_{\alpha}$ such that each $A_{\alpha}$ is $\Gamma_{\beta_{\alpha}}$-complete (here $\Gamma=\Sigma$ or $\Pi$ ) such that one of the following holds:

(1) $\beta_{\alpha}+i=\beta_{\alpha+\delta}$ for some $\delta>\omega$ and $i \leq 2$, or

(2) $\beta_{\alpha}+i=\beta_{\alpha+1}$ for some $i>2$.

It is open whether $A C G$ provides an example satisfying part (2) of the above. Naively, the derivation process producing the classical rank on $A C G$ could require $i=4$.

Question 32. What are the exact descriptive complexities of the sets $A C G_{\alpha}$ ?

Finally, although $\left\{T \in W F:|T|_{l s} \leq \alpha\right\}$ and $\{T \in W F:|T| \leq \omega \alpha\}$ are both $\Sigma_{2 \alpha^{-}}^{0}$ complete, and thus there is a reduction from one to the other that passes through the universal set $\left\{X: n_{0} \in H_{2^{a}}^{X}\right\}$ for appropriate $a$, we are not aware of a natural reduction between them.

Question 33. Give natural computable functions $f$ and $g$ such that $f^{-1}\left(\left\{T:|T|_{l s} \leq \alpha\right\}\right)=$ $\{T:|T| \leq \omega \alpha\}$ and $g^{-1}(\{T:|T| \leq \omega \alpha\})=\left\{T:|T|_{l s} \leq \alpha\right\}$.

\section{REFERENCES}

[AK00] C. J. Ash and J. Knight. Computable structures and the hyperarithmetical hierarchy, volume 144 of Studies in Logic and the Foundations of Mathematics. North-Holland Publishing Co., Amsterdam, 2000.

[CM82] Douglas Cenzer and R. Daniel Mauldin. On the Borel class of the derived set operator. Bull. Soc. Math. France, 110(4):357-380, 1982.

[CM83] Douglas Cenzer and R. Daniel Mauldin. On the Borel class of the derived set operator. II. Bull. Soc. Math. France, 111(4):367-372, 1983.

[DK91] Randall Dougherty and Alexander S. Kechris. The complexity of antidifferentiation. Adv. Math., 88(2):145-169, 1991.

[GMS13] Noam Greenberg, Antonio Montalbán, and Theodore A. Slaman. Relative to any nonhyperarithmetic set. J. Math. Log., 13(1):1250007, 26, 2013.

[HPZvcZ99] P. Holický, S. P. Ponomarev, L. Zají ček, and M. Zelený. Structure of the set of continuous functions with Luzin's property (N). Real Anal. Exchange, 24(2):635-656, 1998/99.

[KW86] Alexander S. Kechris and W. Hugh Woodin. Ranks of differentiable functions. Mathematika, 33(2):252-278 (1987), 1986.

[Lem87] Steffen Lempp. Hyperarithmetical index sets in recursion theory. Trans. Amer. Math. Soc., 303(2):559-583, 1987.

[Sak64] Stanisł aw Saks. Theory of the integral. Second revised edition. English translation by L. C. Young. With two additional notes by Stefan Banach. Dover Publications, Inc., New York, 1964.

[Wal17] Sean Walsh. Definability aspects of the Denjoy integral. Fund. Math., 237(1):1-29, 2017.

[Wes14] Linda Brown Westrick. A lightface analysis of the differentiability rank. J. Symb. Log., 79(1):240-265, 2014. 
Department of Mathematics, Penn State University, University Park, PA, USA

E-mail address: westrick@psu.edu 